This article is a part of the Special Issue on Aquaculture

\title{
Proactive coping style in early emerging rainbow trout carries a metabolic cost with no apparent return ${ }^{\text {मt }}$
}

\author{
Peter Vilhelm Skov*, Kim Joao de Jesus Gregersen, Manuel Gesto, Alfred Jokumsen \\ Technical University of Denmark, DTU Aqua, Section for Aquaculture, The North Sea Science Park, 9850 Hirtshals, Denmark
}

\section{A B S T R A C T}

The timing with which salmonid larvae emerge from their gravel nests is thought to be correlated with a particular suite of behavioural and physiological traits that correspond to the stress coping style of the individual. Among these traits, aggressiveness, dominance and resilience to stress, are potentially interesting to exploit in aquaculture production. In the present study a series of experiments were performed, with the purpose of characterising behavioural, metabolic and production related traits in rainbow trout juveniles from different emergence fractions. Newly hatched rainbow trout were sorted according to their emergence time from an artificial redd. The early, middle, and late fractions were retained and assessed for their physiological response to stress, growth performance, metabolism, fasting tolerance, and potential for compensatory growth. The early emerging fraction showed proactive behavioural traits; they were faster to reappear following startling, showed a reduced cortisol response following stress, and a reduced metabolic cost of recovery. Emergence time was not correlated with any differences in standard or maximum metabolic rates, but was however, correlated with higher routine metabolic rates, as demonstrated by significantly bigger weight losses during fasting in the early emerging group. Growth rates and feed conversion efficiencies were not significantly different when fish were co-habitated under a restrictive feeding regime, suggesting that early emerging fish are not able to monopolise food resources. The intermediate emerging group, which makes up the bulk of a population and is often ignored, appears to possess the best growth performance traits, possibly because they do not expend excessive energy on dominance behaviour such as the early emerging group, while they are also not overly timid or stress prone such as the late emerging group.

\section{Introduction}

The behavioural response of individual fish to stressful or unfamiliar conditions is correlated to their personality (Ruiz-Gomez and Huntingford, 2012; Larsen et al., 2015). On the personality continuum, extreme behavioural types occur at relatively low frequencies, while the majority of a population occupies intermediate positions (Wolf et al., 2007). The terminology applied to the different responses depends large on whether the context is behavioural or physiological (Castanheira et al., 2017), and has been referred to as personality (Francis, 1990), behavioural syndrome (Sih et al., 2004), or stress coping style (Koolhaas et al., 1999).

For salmonids, the stress coping style of an individual has been shown to be correlated with the time of emergence from the gravel spawning nest (Vaz-Serrano et al., 2011; Andersson et al., 2013a). Early emerging individuals have been shown to be bolder and more aggressive (Metcalfe and Thorpe, 1992; Metcalfe et al., 1995), be less sensitive to confinement stress (Larsen et al., 2015), and have shorter feeding latencies and higher feeding rates (Vaz-Serrano et al., 2011), than late emerging individuals. Furthermore, a positive correlation has been shown between boldness and activity levels (Larsen et al., 2015).

Energetically, early emergers have an advantage over late emergers, in that they have earlier access to feed (Brännäs, 1995). Early emerging individuals tend to dominate over late emergers, although this dominance is not correlated with body size, and thereby not a result of early feeding. Instead it has been proposed that the behavioural profile of early emergers is a contributing factor (Metcalfe and Thorpe, 1992).

For some species, a correlation between stress-coping style and metabolic rate has been demonstrated, in which individuals with higher oxygen consumption rates are more likely to display proactive-like (bold, aggressive, and dominant) behaviour. This has been shown for common carp (Cyprinus carpio), where oxygen uptake rates in bold (risk taking) fish were $\sim 15 \%$ higher than for shy (risk avoiding) individuals (Huntingford et al., 2010), as well as for Atlantic salmon (Salmo salar), where individuals displaying a high number of aggressions also had higher metabolic rates (Cutts et al., 1998). Higher standard metabolic rates effectively decrease both scope for activity (Fry, 1947) and growth (Elliott, 1976), unless maximum metabolic rates are correspondingly elevated. If, as proposed by Killen et al. (2012), high standard metabolic rates should encompass the ability to achieve higher rates of growth or activity levels, proactive individuals should also be able to achieve higher maximum metabolic rates. So far, there is little evidence to support this. For $S$. salar, a higher standard metabolic rate comes at the cost of a reduced metabolic scope (Cutts et al., 2002), which in brown

\footnotetext{
This article is part of a special issue entitled: Aquaculture, edited by: Dr. Tillmann Benfey, Dr. Inna Sokolova and Dr. Mike Hedrick.

* Corresponding author.

E-mail address: pvsk@aqua.dtu.dk (P.V. Skov).
} 
trout (S. trutta) has been linked to reduced feed intake (Auer et al., 2015).

In aquaculture, there is a great interest in obtaining lines of robust fish for on-growing, based on the assumption that these are more resilient to stress and disease, feed more and utilize feed more efficiently (Castanheira et al., 2017). Selecting rainbow trout based on their emergence time could therefore be a potential way to obtain fish with improved stress coping ability and higher growth potential. The bioenergetic, territorial and behavioural profiles have, to some extent, been described for early and late emergers (Brännäs, 1995; Larsen et al., 2015). Additionally, some production related studies have been conducted, focusing on how fish from different emergence fractions handle crowding or other stressful conditions (Trenzado et al., 2006; Van de Nieuwegiessen et al., 2010). However, while proactive fish may be better able to handle stressful conditions, relatively little is known about their performance in a production environment under routine conditions - that is, not deliberately subjected to known stressful conditions. The aim of the present study was to test the hypothesis that different emergence fractions were characterised by specific behavioural or physiological traits. We subsequently wanted to examine to what extent these traits influence energetics, feeding behaviour, and growth, i.e. potentially advantageous to select for in aquaculture production. To this end, selected emergence fractions of rainbow trout were subjected to respirometry, behavioural tests, and growth trials, to determine their behavioural and physiological characteristics.

\section{Materials and methods}

\subsection{Research animals and housing}

A total of 10.000 fertilized all-female rainbow trout (Oncorhynchus mykiss) eggs were obtained from Seven Springs Trout Hatchery Ltd. (Batch no. 14053, Co Antrim, North Ireland, United Kingdom). Upon arrival, eggs were 265 degree-days (accumulated temperature unit). Eggs were evenly distributed in 3 tray incubators with screen bottoms supplied by gravity fed water from a reservoir, in a room thermostatted at $7^{\circ} \mathrm{C}$. Hatching began at 356 degree-days and was completed at 384 degree-days. Hatched fry were progressively distributed in three separate emergence incubators as described previously (Vaz-Serrano et al., 2011). Emergence incubators consisted of hatching trays with a layer of golf balls covering the bottom, and an emergence route 'downstream', from which emerging fry could be collected, according to their emergence time; the first $20 \%$ to emerge, the next $20 \%$ to emerge, and so forth. These fractions were designated early, early-middle, middle, middle-late and late. Of these, only the early, middle, and late fractions were used in the present study. Fish from the three retained emergence fractions were divided into triplicate $30 \mathrm{~L}$ tanks ( $\varnothing 40 \mathrm{~cm}, \mathrm{~d} 25 \mathrm{~cm}$ ) and reared until they reached a body mass of approximately $3 \mathrm{~g}$, at which time they were transferred to a triplicate system of $1 \times 1 \mathrm{~m}$ tanks with a water depth of $28 \mathrm{~cm}$. Water supply to the tanks came from a recirculated system thermostatted at $16^{\circ} \mathrm{C}$. Fish were maintained on a size-appropriate commercial trout diet (Performa EX, Aller Aqua, Denmark) on the optimal feeding regime as specified by the manufacturer.

The present work was undertaken as a series of individual experiments, some on individual fishes others on groups of fishes. All experiments were conducted in accordance with Danish and EU legislation (Directive 2010/63/EU) under permission from the Danish Animal Research Authority (permit no. 2014-15-0201-00416).

\subsection{Experimental procedures}

\subsubsection{Juvenile growth trial}

To assess overall growth performance of the different emergence fractions, a 7 week growth trial was performed on triplicate groups from each emergence fraction. Each tank $(1 \times 1 \times 0.45 \mathrm{~m})$ was stocked with a biomass of $1200 \mathrm{~g}$ of juvenile fish, with an average body mass of $12.6 \mathrm{~g}$. Fish were fed daily rations corresponding to $2.2 \%$ of their biomass using mechanical belt feeders. Daily rations were adjusted using an assumed feed conversion ratio of 0.7. An intermediate weighing was conducted on day 26, at which time feeding tables were adjusted for observed growth, and again at the end of the growth trial. Feed was withheld for $24 \mathrm{~h}$ prior to weighing to allow gastric evacuation.

\subsubsection{Behavioural response to startling and the presence of a novel object}

In order to standardize the assessment of the behavioural response to a disturbance, a protocol based on the methodology described by Killen et al. (2012) with some modifications was employed. The tests were conducted in a system of 3 identical grey tanks (L $75 \times \mathrm{W} 50 \times \mathrm{H}$ $15 \mathrm{~cm}$ ) that served as behavioural arenas. The end of each arena was fitted with a sheltered area in the form of a Styrofoam sheet covering the first $20 \mathrm{~cm}$ of the tank length and spanning the full width. The remaining $55 \mathrm{~cm}$ was an open arena. Each arena was supplied by a flow of aerated water thermostatted at $16{ }^{\circ} \mathrm{C}$, delivered from a $20 \mathrm{~L}$ sump below, at a rate of $300 \mathrm{Lh}^{-1}$. Water flow was in the direction from the open arena towards the sheltered area. Each arena was visually isolated with opaque plastic sheeting to avoid disturbance, and behavioural activity was video recorded (HD-4110, Hewlett Packard) for later analysis. Fish to be used in experiments were isolated in $30 \mathrm{~L}$ sections of glass aquaria $36 \mathrm{~h}$ prior to behavioural tests. During this time they were fasted to avoid any confounding effects of digestion. On the day of the experiment, a fish from the early, middle and late fraction were assigned randomly to each of the behavioural arenas.

At the beginning of the experiment, the fish were placed in their designated tank and left to acclimatize for $4 \mathrm{~h}$. The trial period lasted $60 \mathrm{~min}$, and started at the end of the $4 \mathrm{~h}$ acclimatization period. The test was initiated by dropping a $3 \times 10 \mathrm{~cm}$ cylinder into the arena at a time when the fish was occupying a part of the open arena. The cylinder was attached to a string, ensuring that it remained hanging in the water column, and that it was always delivered from the same height, and into the same area of the open arena.

The response to the dropping cylinder was for the fish to seek shelter under the Styrofoam cover. In the assessment of fish behaviour, the following 4 parameters were analysed; whether or not the tested fish would re-appear from shelter during the $60 \mathrm{~min}$ observation period, the time it took until it appeared from under the cover (snout of the fish being visible), the time it took for the fish to completely emerge from under the cover, and the time it spent in the open part of the arena. A total of 21-24 fish with similar masses were tested from each fraction (mean body mass $37.1 \pm 1.4 \mathrm{~g}$ ). In the calculation of temporal variables, fish that did not appear from the shelter were excluded from the analyses.

\subsubsection{Respiratory physiology and post-stress oxygen debt}

Following the $60 \mathrm{~min}$ observation in the behavioural experiment fish were subjected to a chase protocol as described by Norin and Malte (2011). Individual fish were placed in a circular tub $(\varnothing 40 \mathrm{~cm})$ containing $40 \mathrm{~L}$ aerated water at $16^{\circ} \mathrm{C}$, and chased by hand until they did not respond to handling. Fish were then transferred to an individual respirometer in a triplicate system. Respirometers were immersed in a $235 \mathrm{~L}$ bath receiving aerated, UV treated water from a $600 \mathrm{~L}$ reservoir thermostatted at $16^{\circ} \mathrm{C}$, with a daily water replacement of $40 \%$. The volume of each respirometer, including the recirculation loop, was $950 \mathrm{~mL}$. Oxygen consumption was determined using intermittent flowthrough respirometry as described by Steffensen (1989), using $840 \mathrm{~s}$ loops, consisting of a $60 \mathrm{~s}$ wait period, a $500 \mathrm{~s}$ measurement period, and a $280 \mathrm{~s}$ flush period. Oxygen content was measured using fibre optic oxygen sensors (Oxy-4 mini, PreSens Precision Sensing GmbH, Regensburg, Germany). Oxygen data was collected over a $24 \mathrm{~h}$ period, and analysed online using automated software (AutoResp2, Loligo Systems, Tjele, Denmark). Respirometers were covered by opaque plastic during measurements to avoid visual disturbance. The first oxygen 
consumption measurement $\left(\mathrm{MO}_{2}\right)$ following transfer to the chamber was taken as the maximum oxygen consumption rate $\left(\mathrm{MO}_{2}\right.$ MAX). Standard metabolic rates (SMR) were calculated from a frequency distribution of all measurements as described by Skov et al. (2011). Metabolic scope was calculated in absolute terms, as the difference between SMR and $\mathrm{MO}_{2}$ MAX. Fish were considered to have repaid their oxygen debt following stress when 2 consecutive $\mathrm{MO}_{2}$ measurements were within $10 \%$ of the calculated SMR. Oxygen debt was calculated as the summated $\mathrm{MO}_{2}$ within this time period.

\subsubsection{Cortisol response to stress}

In order to determine the magnitude of the cortisol release following stress, 8 fish from the early $(60.0 \pm 2.2 \mathrm{~g})$, middle $(62.1 \pm 2.7 \mathrm{~g})$ and late $(61.0 \pm 3.3 \mathrm{~g})$ emergence fraction, were isolated in $30 \mathrm{~L}$ sections of glass aquaria, and allowed to acclimate overnight. The following day, each fish was individually netted and anaesthetised in $40 \mathrm{mg} \mathrm{L}^{-1}$ benzocaine, while taking care not to disturb other fish. Upon loss of equilibrium, a $300 \mu \mathrm{L}$ blood sample was taken. Fish were then recovered from anaesthesia, and subjected to a 5 min stress challenge where the water level was lowered so that fish were forced to lie on their side. At the end of the challenge, approximately $10 \mathrm{~min}$ after first being netted from the tank, fish were re-anaesthetised, and an additional $300 \mu \mathrm{L}$ blood sample was drawn. Blood was centrifuged at $10000 \mathrm{~g}$ at $4{ }^{\circ} \mathrm{C}$ for 5 min immediately after being sampled. The plasma fraction was then transferred to a cryovial, frozen on dry ice, and stored at $-80^{\circ} \mathrm{C}$ until analysis. Following ethyl ether extraction, plasma samples were analysed for cortisol using a commercial ELISA kit (Neogen Europe Ltd.) according to the protocol provided by the manufacturer.

\subsubsection{Fasting and re-feeding in individual fish}

To assess the rate of weight loss during periods of feed limitation, and their potential for weight gain during re-feeding, 10 fish from the early $(57.4 \pm 1.7 \mathrm{~g})$, middle $(51.9 \pm 1.4 \mathrm{~g})$, and late $(51.6 \pm 2.2 \mathrm{~g})$ emergence fraction were individually isolated in $30 \mathrm{~L}$ sections of glass aquaria. Fish that had been fasted for $24 \mathrm{~h}$ were weighed upon insertion. Feed was then withheld for a period of 10 days, after which fish were re-weighed. The following 7 days, fish were fed ad libitum for $1 \mathrm{~h}$ each morning. Any uneaten feed was siphoned from the tanks at the end of the feeding period. After 7 days of re-feeding, fish were fasted for $24 \mathrm{~h}$ and weighed again. Rates of weight loss and weight gain were calculated as specific growth rates (SGR, \% $\mathrm{d}^{-1}$ ).

\subsubsection{Fasting and re-feeding during co-habitation}

To assess whether rates of weight loss and weight gain during semirestrictive re-feeding were affected by co-habitation of different emergence fractions, a total of 75 fish from the early $(67.7 \pm 0.6 \mathrm{~g})$, middle $(68.1 \pm 0.9 \mathrm{~g})$, and late $(65.1 \pm 1.3 \mathrm{~g})$ fraction were tagged with passive integrated transponders (PIT tag, $2 \times 12 \mathrm{~mm}$ ), and 25 fish from each fraction were stocked in triplicate in $450 \mathrm{~L}$ tanks $(1 \times 1 \times 0.45 \mathrm{~m})$ connected to a recirculating system thermostatted at $16^{\circ} \mathrm{C}$. Fish were allowed to recover from tagging for a period of 2 weeks during which time they were fed a daily ration corresponding to $1.5 \%$ of their body mass. After recovery, fish were weighed again, returned to the tanks and fasted for 10 days. Fish were then re-weighed, and fed a restricted ration of $0.9 \%$ per day for the following 21 days, fasted for $24 \mathrm{~h}$ and reweighed.

\subsection{Statistical analyses}

All data are presented as mean values \pm standard error of the mean (SEM). Statistical analyses were performed using SigmaPlot 13 (Systat Software, Inc., San Jose, CA). Differences in the proportion of fish from the different emergence fractions to appear following the startle trial were assessed using a Chi-square for equality of proportions. All other data was subjected to a one-way ANOVA after being tested for normality and equal variance. In all cases, statistical significance was
Table 1

Initial growth of early, middle and late emerging fractions. Data are mean values from triplicate tanks, and based on a 7 week growth trial, feeding at a daily rate corresponding to $2.2 \%$ of the tank biomass. Feed conversion ratio (FCR) is calculated as unit of feed intake per unit of growth. Specific growth rate (SGR) is calculated as (Ln (Final mass) - Ln (Initial mass)) / number of feeding days $\times$ $100 \%$.

\begin{tabular}{lllll}
\hline & Early & Middle & Late & $p$ \\
\hline Initial mass $(\mathrm{g})$ & $14.2 \pm 1.1$ & $11.6 \pm 0.5$ & $12.1 \pm 0.7$ & 0.125 \\
Final mass $(\mathrm{g})$ & $54.3 \pm 2.2$ & $48.0 \pm 1.2$ & $49.5 \pm 1.6$ & 0.092 \\
Weight gain $(\mathrm{g})$ & $40.2 \pm 1.1$ & $36.5 \pm 0.7$ & $37.4 \pm 1.0$ & 0.075 \\
FCR $\left(\mathrm{g} \mathrm{feed}^{-1}\right.$ ) & $0.80 \pm 0.01$ & $0.80 \pm 0.00$ & $0.80 \pm 0.01$ & 0.879 \\
SGR $\left(\%\right.$ day $^{-1}$ ) & $2.28 \pm 0.11$ & $2.50 \pm 0.05$ & $2.45 \pm 0.07$ & 0.196 \\
\hline
\end{tabular}

accepted at $p<.05$.

\section{Results}

\subsection{Initial growth}

Results from the initial growth trial are given in Table 1. Similar weight gains, feed conversion ratios and specific growth rates were observed for all 3 emergence fractions.

\subsection{Behavioural response to startling and the presence of a novel object}

All fish, regardless of their emergence fraction, responded to startling by seeking refuge under the sheltered area of the tank. There were significant differences in the proportion of fish that reappeared during the $60 \mathrm{~min}$ that the trial lasted $(p=.032)$. From the early emerging fraction, 16 out of 23 tested fish (69.6\%) re-appeared within the $60 \mathrm{~min}$ observation period. From the middle fraction, 9 out of 22 (40.9\%) reappeared, and from the last fraction, 6 out of 18 (33.3\%) reappeared. There were no significant differences between groups in the time it took to first appear from under the shelter $(p=.298)$, to fully emerge $(p=.591)$, or the total time spent in the open part of the arena $(p=.772)$.

\subsection{Respiratory physiology and post-stress oxygen consumption}

Early, middle, and late emerging fractions of rainbow trout showed no differences in their SMR $(p=.735)$, MMR $(p=.399)$ or metabolic scope $(p=.123)$ (Table 2$)$. The late emerging fraction had a post-stress oxygen debt that was $55-70 \%$ higher $(p=.005)$ than that of the middle and early emerging fractions (Fig. 1B). With no differences in MMR between fractions, this resulted in significantly longer $(p=.001)$ recovery times for the late emerging fraction (Fig. 1C).

\subsection{Stress response}

No differences in resting cortisol levels were observed between the different emergence fractions (Fig. 1A). The cortisol response following stress was significantly higher $(p=.028)$ in the late emerging fraction than in the early emerging fraction. The middle fraction showed an

Table 2

Mass specific standard (SMR) and maximum (MMR) metabolic rates of early $(n=7)$, middle $(n=9)$ and late $(n=7)$ emerging fish. Metabolic scope (MS) is calculated in absolute terms as MMR less SMR.

\begin{tabular}{lllll}
\hline & Early & Middle & Late & $p$ \\
\hline SMR $\left(\mathrm{mg} \mathrm{O}_{2} \mathrm{~kg}^{-1} \mathrm{~h}^{-1}\right)$ & $169.6 \pm 10.4$ & $169.5 \pm 6.7$ & $166.7 \pm 9.6$ & 0.966 \\
${\mathrm{MMR}\left(\mathrm{mg} \mathrm{O}_{2}\right.}_{\left.\quad \mathrm{kg}^{-1} \mathrm{~h}^{-1}\right)}^{553.5 \pm 27.7}$ & $533.5 \pm 21.2$ & $525.0 \pm 24.8$ & 0.711 \\
$\mathrm{MS}\left(\mathrm{mg} \mathrm{O}_{2} \mathrm{~kg}^{-1} \mathrm{~h}^{-1}\right)$ & $384.0 \pm 31.0$ & $364.0 \pm 17.7$ & $358.4 \pm 21.2$ & 0.732
\end{tabular}



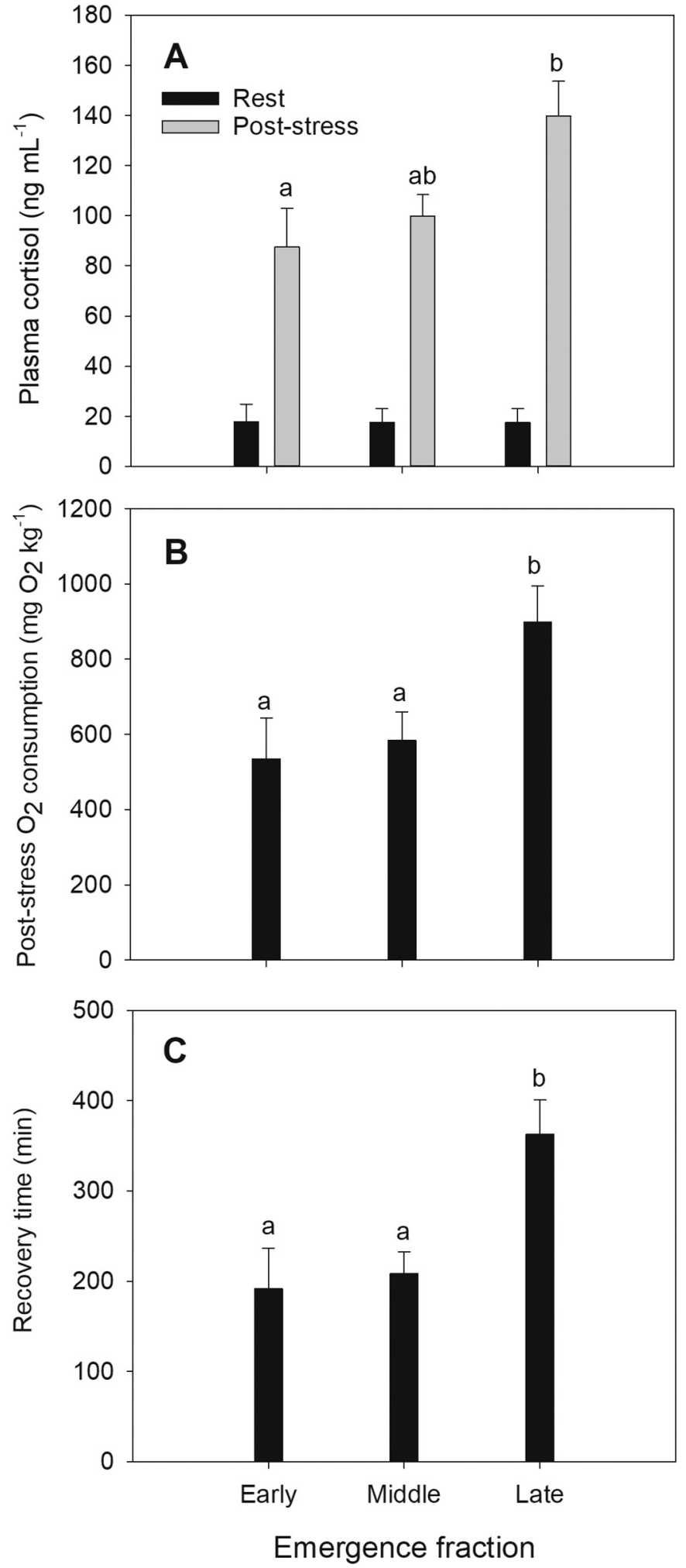

Fig. 1. Baseline and post-stress cortisol levels in individuals from of the early $(\mathrm{n}=9)$, middle $(n=10)$, and late $(\mathrm{n}=9)$ emergence fractions are shown in (a), the cumulative oxygen debt (summated oxygen consumption in excess of SMR) during recovery from stress (b), and the total time taken to recover from stress as quantified by the oxygen debt being repaid (c). intermediate cortisol response that was not significantly different from the early or late fraction.

\subsection{Fasting and re-feeding in single fish}

There were no differences in initial body masses prior to the experiment $(p=.751)$. Absolute losses, gains and growth rates during 10 days of fasting and 7 days of refeeding of individual fish are given in Table 3. Weight loss during fasting, was significantly higher in the early emerging fraction, than in the late emerging fraction. Early and late emerging fractions displayed significantly lower SGRs during re-feeding than the intermediate group (Fig. 2). The ratio between weight gain during re-feeding, and weight loss during fasting, was significantly higher in the intermediate individuals than in the early emerging fraction. The intermediate group regained lost body mass 5.7-fold, while the early and late emerging fraction only achieved 3.6 and 4.9 fold gains in body mass. Feed intake during re-feeding was not recorded; therefore it was not possible to calculate feed conversion efficiency.

\subsection{Fasting and re-feeding during co-habitation}

Weight losses and gains in the co-habitation experiment are shown in Fig. 2.There were no significant differences in weight losses during fasting. These ranged from $3.38 \pm 0.19 \mathrm{~g}$ in the late emerging fraction $(5.29 \pm 0.21 \% \mathrm{BM})$ to $3.74 \pm 0.06 \mathrm{~g}(5.58 \pm 0.04 \%)$ in the early emerging fraction. The intermediate group performed best, with intermediate weight losses during fasting, and the highest weight gains during refeeding. The intermediate group achieved the highest gain to loss ratio for the three groups, at $3.75 \pm 0.07 \mathrm{~g}$ gained per $\mathrm{g}$ lost.

\section{Discussion}

The behavioural assessment of rainbow trout in the present study confirms that different emergence fractions possess some of the physiological and behavioural traits that are characteristic of the reactive and proactive coping styles. The behavioural experiments and oxygen consumption measurements during recovery from chasing suggest that fish from the early emerging fraction are bolder and less stress sensitive than the late emerging fractions. This was further supported by the cortisol measurements following stress exposure. Weight loss during starvation was used as a proxy for energy use, and showed that fish from the early emerging fraction have higher routine metabolic rates than the late emerging individuals. In a number of other physiological aspects, such as resting cortisol levels, SMR and MMR, the emergence fractions did not differ.

The behavioural response to an object dropping into the holding tank, was identical for all emergence fractions. From the early emerging fraction, a significantly higher proportion of fish reappeared during the observation period. This suggests that fish from the early emerging fraction are bolder and more exploratory. However, regardless of emergence fraction, there were no differences in the time it took fish to reappear, or the amount of time they spent in the open arena during the observation period. Our observations support earlier findings by RuizGomez et al. (2008), who showed that dominant rainbow trout startled by a novel object are quicker to resume previous behaviour. The lack of difference in the time spent in the open end of the arena are in contrast to the results by Sneddon (2003). Here, bold rainbow trout spent the majority of their time in the open part of an arena, while shy individuals preferred to stay under shelter. The presence of a feeding stimulus in the study by Sneddon (2003) may have been an influencing factor, but as may other factors such as overhead fluorescent lighting or colour of the test tank.

A more proactive personality in the early emerging fraction was further supported by the stress and recovery experiments. Proactive individuals usually have a lower reactivity of the hypothalamus- 
Table 3

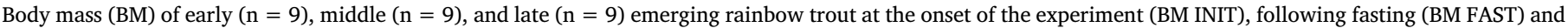

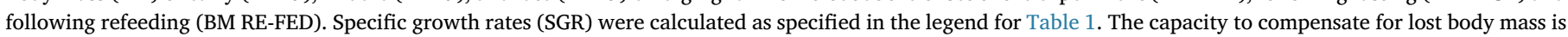
expressed as the ratio between weight gain and weight loss (gain:loss abs).

\begin{tabular}{|c|c|c|c|c|}
\hline & Early & Middle & Late & $\mathrm{p}$ \\
\hline BM INIT (g) & $53.41 \pm 1.67$ & $51.93 \pm 1.35$ & $51.63 \pm 2.19$ & 0.751 \\
\hline BM FAST (g) & $50.21 \pm 1.56$ & $49.31 \pm 1.26$ & $49.30 \pm 2.11$ & 0.908 \\
\hline BM RE-FED (g) & $61.29 \pm 1.86$ & $64.02 \pm 1.94$ & $60.44 \pm 2.93$ & 0.524 \\
\hline SGR FAST $\left(\% \mathrm{~d}^{-1}\right)$ & $-0.62 \pm 0.04^{a}$ & $-0.52 \pm 0.03^{\mathrm{ab}}$ & $-0.46 \pm 0.04^{b}$ & 0.011 \\
\hline SGR RE-FEED $\left(\% \mathrm{~d}^{-1}\right)$ & $2.00 \pm 0.15$ & $2.60 \pm 0.14$ & $2.02 \pm 0.25$ & 0.050 \\
\hline BM abs loss (g) & $3.20 \pm 0.23^{\mathrm{a}}$ & $2.62 \pm 0.15^{\mathrm{ab}}$ & $2.33 \pm 0.20^{b}$ & 0.013 \\
\hline BM relative loss (\%) & $5.98 \pm 0.35^{\mathrm{a}}$ & $5.04 \pm 0.24^{\mathrm{ab}}$ & $4.53 \pm 0.35^{b}$ & 0.011 \\
\hline BM abs gain $(\mathrm{g})$ & $11.08 \pm 0.95$ & $14.71 \pm 1.00$ & $11.14 \pm 1.52$ & 0.065 \\
\hline BM relative gain (\%) & $22.19 \pm 1.87^{\mathrm{a}}$ & $29.80 \pm 1.74^{b}$ & $22.71 \pm 3.03^{\mathrm{a}}$ & 0.048 \\
\hline Gain:Loss abs & $3.55 \pm 0.33^{a}$ & $5.74 \pm 0.44^{b}$ & $4.86 \pm 0.56^{\mathrm{ab}}$ & 0.008 \\
\hline
\end{tabular}

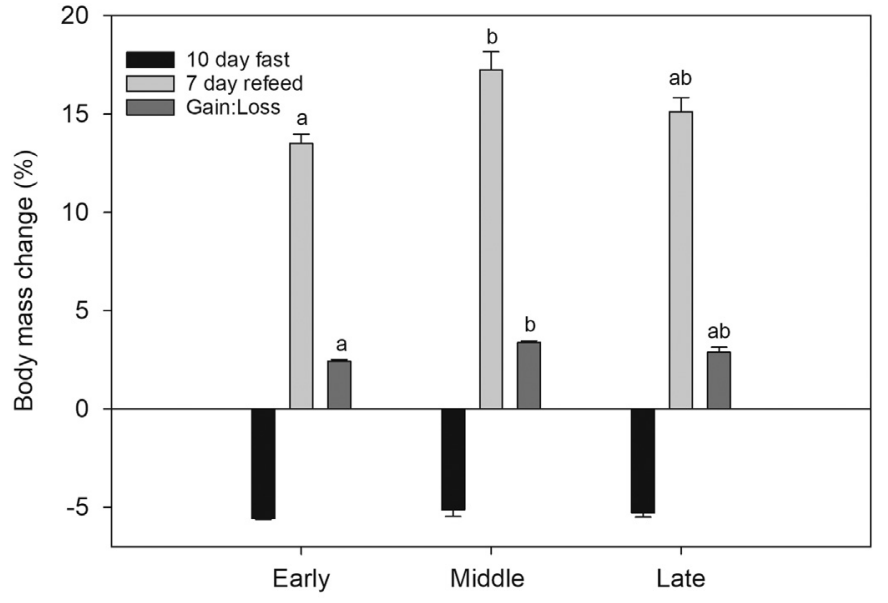

\section{Emergence fraction}

Fig. 2. Changes in body mass following fasting and re-feeding in the 3 emergence fraction, during co-habitation. Values are means of triplicate groups. Changes in body mass are expressed in relative terms (\%).

pituitary-interrenal (HPI) axis upon stress exposure than reactive individuals (Koolhaas et al., 2010; Réale et al., 2007; Silva et al., 2010; Vindas et al., 2017). During undisturbed conditions, plasma cortisol levels did not differ between emergence fractions, suggesting that activity levels of the HPI axis are similar. Reactivity of the HPI axis on the other hand, differed significantly between emergence fractions. Following the manual chase protocol, the plasma cortisol levels in the late emerging fraction reached $140 \mathrm{ng} \mathrm{mL}^{-1}$, whereas the early and middle fraction increased to $90-100 \mathrm{ng} \mathrm{mL}^{-1}$. These levels correlate with the results of Pottinger and Carrick (1999) who selected rainbow trout based on their HPI reactivity. In these fish, low and high responsive fish had cortisol levels of ca. 80 and $180 \mathrm{ng} \mathrm{mL}^{-1}$, respectively. The low reactivity of the HPI axis supports the hypothesis that early emerging fish are more proactive. The reduced HPI reactivity allowed the early emerging fish to recover from a stressful event significantly faster than the late emerging group ( $196 \pm 31 \mathrm{~min}$ v. $408 \pm 32 \mathrm{~min})$ while the intermediate emerging fraction was in between (319 \pm 31$)$. Since all emergence fractions repaid their oxygen debt at the same rate, between 0.47 and $0.50 \mathrm{~min} \mathrm{mg}^{-1} \mathrm{O}_{2}$ debt, the faster recovery of the early emerging fraction was the result of a proportionally smaller oxygen debt following stress.

The absence of any differences in standard and maximum metabolic rates between emergence fractions is in accordance with our previous observations from $S$. salar (Vaz-Serrano et al., 2011), and from rainbow trout that originated from a population selectively bred for their post stress cortisol levels (Moltesen et al., 2016). While we were unable to detect differences in SMR in the present study, it has been shown that dominance in individuals of $S$. salar is correlated with higher standard metabolic rates (SMR) than subordinate individuals and that this dominance is found in first feeding (e.g. early emerging) individuals (Metcalfe et al., 1995). These authors suggest, that a high SMR is associated with a greater metabolic scope and energetic capacity for acts of aggression. This is in accordance with the performance model of metabolic rate (Careau et al., 2008), in which animals with high metabolic rates can collect, process, and invest nutritional energy faster, and therefore achieve higher growth rates and afford higher activity levels. While a greater metabolic scope would allow for the potential increased growth, while still accommodating the metabolic cost of aggression, this did not appear to apply to the early emergence fraction in the present study.

Intuitively, individuals with a high routine metabolism may be driven to process more feed on a daily basis to fuel this elevated RMR. As pointed out by Careau et al. (2008) this may lead to hypertrophy of the digestive organs. Because digestive organs of fish may have a mass specific metabolism that is 25-35 times higher than e.g. skeletal muscle (Oikawa and Itazawa, 2003), increased feed intake drives an increase in standard metabolic rate. The causality between feeding level, hypertrophy of digestive tissues, and metabolism, warrants further investigation.

During the past years, several methods to correlate metabolic rates with personality or coping style in fishes have been used. Although standard (SMR) and routine metabolic rates (RMR) are occasionally used interchangeably, routine metabolic rate has a behavioural component. It includes the metabolic cost of voluntary activity, and is a much more likely measure to reflect the metabolic cost of personality. While we did not quantify RMR directly (i.e. by respirometry), it is clear from the weight loss during fasting, that the early emerging fraction had significantly higher rates of substrate utilization to fuel metabolism. This suggests that there are metabolic costs associated with the proactive behavioural profile. That a similar correlation between RMR and weight loss during fasting has been shown in European sea bass (Dicentrarchus labrax) (Killen et al., 2011), lends support to this suggestion.

A higher RMR must drive an increase in feed intake, and it was therefore hypothesised that the early emerging fraction to some extent would monopolise feed resources during co-habitation, and that this might yield superior growth. Proactive individuals are more likely to actively forage, whereby they are able to achieve higher growth rates, even when resources are limited (Hoogenboom et al., 2013; Reid et al., 2012). Contrary to expectations, when trout from the early, middle and late fraction were co-habitated, the middle fraction showed superior performance to the late and early emerging fraction. One possible explanation for why early emerging fish in the present experiment did not show superior growth, could be the presence of a large number of conspecifics. This may create the equivalent of a complex environment, whereby fish lose their advantage (Höjesjö et al., 2004). However, data 
from refeeding single fish also failed to demonstrate an increased capacity of early emergers to assimilate feed.

Boldness plays an ecological role when individuals compete for the same resource (Ioannou et al., 2008), but in the present study, this was not clear. The present study showed that the early emerging fraction of rainbow trout show characteristics of having proactive coping styles, but under the conditions of the present experiment, an obvious advantage of being proactive was not apparent. In fact, the middle emerging fraction, which is assumed to be the general representative of a population (Wolf et al., 2007), performed superiorly. Interestingly, it appears that the middle fraction possesses the best mix of characteristics; it has the behavioural reluctance of the late emerging fraction, the reduced stress sensitivity of the early emergers, and an intermediate routine metabolism. The overall result is an increased willingness to feed, which lends support to earlier work on juvenile rainbow trout (Andersson et al., 2013b).

The present results on growth performance were obtained from fish reared in a controlled, and presumably low-stress environment, in which a constant temperature was maintained, oxygen levels were kept near saturation, and fish were not crowded. Under these conditions, the early emerging fraction did not show superior performance, but it cannot be ruled out that the boldness and reduced HPI reactivity of the early emerging fraction may be advantageous in other situations. Although we have shown that the rainbow trout in the present study show distinct behavioural and physiological phenotypes that correlate with emergence time, it is important to note that the genetic background of the fish is an important factor influencing the relationship between emergence time and the stress coping style. In a recent study, Gesto et al. (2017) we showed that the emergence time did not correlate with the stress coping style in a population of rainbow trout that had been selectively bred for over 20 generations. It is possible that the selective pressure in breeding may diminish the phenotype diversity in terms of boldness/shyness, contributing to a higher behavioural homogeneity of the fish.

\section{Acknowledgements}

The present research was conducted as part of the project "RobustFish" funded by the Danish Agrifish Agency through the Green Development and Demonstration Programme (Grant no. 34009-130677), and the International Centre for Research in Organic Food Systems (ICROFS, Denmark).

\section{References}

Andersson, M.Å., Khan, U.W., Øverli, T., Gjøen, H.M., Höglund, E., 2013a. Coupling between stress coping style and time of emergence from spawning nests in salmonid fishes: Evidence from selected rainbow trout strains (Oncorhynchus mykiss). Physiol. Behav. 116-117, 30-34. https://doi.org/10.1016/j.physbeh.2013.03.019.

Andersson, M.Å., Laursen, D.C., Silva, P.I.M., Höglund, E., 2013b. The relationship between emergence from spawning gravel and growth in farmed rainbow trout Oncorhynchus mykiss. J. Fish Biol. 83, 214-219. https://doi.org/10.1111/jfb.12153.

Auer, S.K., Salin, K., Anderson, G.J., Metcalfe, N.B., 2015. Aerobic scope explains individual variation in feeding capacity. Biol. Lett. 11, 20150793. https://doi.org/10. 1098/rsbl.2015.0793.

Brännäs, E., 1995. First access to territorial space and exposure to strong predation pressure: A conflict in early emerging Atlantic salmon (Salmo salar L.) fry. Evol. Ecol. 9, 411-420. https://doi.org/10.1007/BF01237763.

Careau, V., Thomas, D., Humphries, M.M., Réale, D., 2008. Energy metabolism and animal personality. Oikos 117, 641-653. https://doi.org/10.1111/j.0030-1299.2008. 16513.x.

Castanheira, M.F., Conceição, L.E.C., Millot, S., Rey, S., Bégout, M.-L., Damsgård, B., Kristiansen, T., Höglund, E., Øverli, Ø., Martins, C.I.M., 2017. Coping styles in farmed fish: Consequences for aquaculture. Rev. Aquac. 9, 23-41. https://doi.org/10.1111/ raq. 12100.

Cutts, C.J., Metcalfe, N.B., Taylor, A.C., 1998. Aggression and growth depression in juvenile Atlantic salmon: The consequences of individual variation in standard metabolic rate. J. Fish Biol. 52, 1026-1037. https://doi.org/10.1006/jfbi.1998.0647.

Cutts, C.J., Metcalfe, N.B., Taylor, A.C., 2002. Juvenile Atlantic salmon (Salmo salar) with relatively high standard metabolic rates have small metabolic scopes. Funct. Ecol. 16, 73-78. https://doi.org/10.1046/j.0269-8463.2001.00603.x.

Elliott, J.M., 1976. The energetics of feeding, metabolism and growth of brown trout
(Salmo trutta L.) in relation to body weight, water temperature and ration size. J. Anim. Ecol. 45, 923-948.

Francis, R.C., 1990. Temperament in a fish: A longitudinal study of the development of individual differences in aggression and social rank in the midas cichlid. Ethology 86, 311-325. https://doi.org/10.1111/j.1439-0310.1990.tb00439.x.

Fry, F.E.J., 1947. Effect of the environment on animal activity. Univ. Toronto Stud. Biol. Ser. 55, 1-62.

Gesto, M., Skov, P.V., Jokumsen, A., 2017. Emergence time and skin melanin spot patterns do not correlate with growth performance, social competitive ability or stress response in farmed rainbow trout. Front. Neurosci. 11, 319. https://doi.org/10. 3389/fnins.2017.00319.

Höjesjö, J., Johnsson, J., Bohlin, T., 2004. Habitat complexity reduces the growth of aggressive and dominant brown trout (Salmo trutta) relative to subordinates. Behav. Ecol. Sociobiol. 56, 286-289.

Hoogenboom, M.O., Armstrong, J.D., Groothuis, T.G.G., Metcalfe, N.B., 2013. The growth benefits of aggressive behavior vary with individual metabolism and resource predictability. Behav. Ecol. 24, 253-261. https://doi.org/10.1093/beheco/ars161.

Huntingford, F.A., Andrew, G., Mackenzie, S., Morera, D., Coyle, S.M., Pilarczyk, M., Kadri, S., 2010. Coping strategies in a strongly schooling fish, the common carp Cyprinus carpio. J. Fish Biol. 76, 1576-1591. https://doi.org/10.1111/j.1095-8649. 2010.02582.x.

Ioannou, C.C., Payne, M., Krause, J., 2008. Ecological consequences of the bold-shy continuum: The effect of predator boldness on prey risk. Oecologia 157, 177-182. https://doi.org/10.1007/s00442-008-1058-2.

Killen, S.S., Marras, S., Mckenzie, D.J., 2011. Fuel, fasting, fear: routine metabolic rate and food deprivation exert synergistic effects on risk-taking in individual juvenile European sea bass. J. Anim. Ecol. 80, 1024-1033. https://doi.org/10.1111/j.13652656.2011.01844.x.

Killen, S.S., Marras, S., Ryan, M.R., Domenici, P., Mckenzie, D.J., 2012. A relationship between metabolic rate and risk-taking behaviour is revealed during hypoxia in juvenile European sea bass. Funct. Ecol. 26, 134-143. https://doi.org/10.1111/j.13652435.2011.01920.x.

Koolhaas, J.M., Korte, S.M., De Boer, S.F., Van Der Vegt, B.J., Van Reenen, C.G., Hopster, H., De Jong, I.C., Ruis, M.A.W., Blokhuis, H.J., 1999. Coping styles in animals: Current status in behavior and stress- physiology. Neurosci. Biobehav. Rev. 23, 925-935. https://doi.org/10.1016/S0149-7634(99)00026-3.

Koolhaas, J.M., de Boer, S.F., Coppens, C.M., Buwalda, B., 2010. Neuroendocrinology of coping styles: towards understanding the biology of individual variation. Front. Neuroendocrinol. 31, 307-321.

Larsen, M.H., Johnsson, J.I., Winberg, S., Wilson, A.D.M., Hammenstig, D., Thörnqvist, P.O., Midwood, J.D., Aarestrup, K., Höglund, E., 2015. Effects of emergence time and early social rearing environment on behaviour of Atlantic salmon: Consequences for juvenile fitness and smolt migration. PLoS One 10 (3). https://doi.org/10.1371/ journal.pone.0119127. ISSN 1932-6203.

Metcalfe, N.B., Thorpe, J.E., 1992. Early predictors of life-history events: The link between first feeding date, dominance and seaward migration in Atlantic salmon, Salmo salar L. J. Fish Biol. 41, 93-99. https://doi.org/10.1111/j.1095-8649.1992. tb03871.x.

Metcalfe, N.B., Taylor, A.C., Thorpe, J.E., 1995. Metabolic rate, social status and life history strategies in Atlantic salmon. Anim. Behav. 49, 431-436. https://doi.org/10. 1006/anbe.1995.0056.

Moltesen, M., Vindas, M.A., Winberg, S., Ebbesson, L., De, Lourdes, Ruiz-Gomez, M., Skov, P.V., Dabelsteen, T., Øverli, Ø., Höglund, E., 2016. Cognitive appraisal of aversive stimulus differs between individuals with contrasting stress coping styles; evidences from selected rainbow trout (Oncorhynchus mykiss) strains. Behaviour 153, 1567-1587. https://doi.org/10.1163/1568539X-00003405.

Norin, T., Malte, H., 2011. Repeatability of standard metabolic rate, active metabolic rate and aerobic scope in young brown trout during a period of moderate food availability. J. Exp. Biol. 214, 1668-1675. https://doi.org/10.1242/jeb.054205.

Oikawa, S., Itazawa, Y., 2003. Relationship between summated tissue respiration and body size in a marine teleost, the porgy Pagrus major. Fish. Sci. 69, 687-694. https:// doi.org/10.1046/j.1444-2906.2003.00675.x.

Pottinger, T.G., Carrick, T.R., 1999. Modification of the plasma cortisol response to stress in rainbow trout by selective breeding. Gen. Comp. Endocrinol. 116, 122-132. https://doi.org/10.1006/gcen.1999.7355.

Réale, D., Reader, S.M., Sol, D., McDougall, P.T., Dingemanse, N.J., 2007. Integrating animal temperament within ecology and evolution. Biol. Rev. 82, 291-318. https:// doi.org/10.1111/j.1469-185X.2007.00010.x.

Reid, D., Armstrong, J.D., Metcalfe, N.B., 2012. The performance advantage of a high resting metabolic rate in juvenile salmon is habitat dependent. J. Anim. Ecol. 81, 868-875. https://doi.org/10.1111/j.1365-2656.2012.01969.x.

Ruiz-Gomez, M.L., Huntingford, F.A., 2012. Boldness and aggressiveness in early and late hatched three-spined sticklebacks Gasterosteus aculeatus. J. Fish Biol. 81, 966-976. https://doi.org/10.1111/j.1095-8649.2012.03340.x.

Ruiz-Gomez, M. de L., Kittilsen, S., Höglund, E., Huntingford, F.A., Sørensen, C., Pottinger, T.G., Bakken, M., Winberg, S., Korzan, W.J., Øverli, Ø., 2008. Behavioral plasticity in rainbow trout (Oncorhynchus mykiss) with divergent coping styles: When doves become hawks. Horm. Behav. 54, 534-538. https://doi.org/10.1016/j.yhbeh. 2008.05.005.

Sih, A., Bell, A., Johnson, J.C., 2004. Behavioral syndromes: An ecological and evolutionary overview. Trends Ecol. Evol. 19, 372-378. https://doi.org/10.1016/j.tree. 2004.04.009.

Silva, P.I.M., Martins, C.I.M., Engrola, S., Marino, G., Øverli, Ø., Conceição, L.E.C., 2010. Individual differences in cortisol levels and behaviour of Senegalese sole (Solea senegalensis) juveniles: Evidence for coping styles. Appl. Anim. Behav. Sci. 124, 75-81. https://doi.org/10.1016/j.applanim.2010.01.008. 
Skov, P.V., Larsen, B.K., Frisk, M., Jokumsen, A., 2011. Effects of rearing density and water current on the respiratory physiology and haematology in rainbow trout, Oncorhynchus mykiss at high temperature. Aquaculture 319, 446-452. https://doi. org/10.1016/j.aquaculture.2011.07.008.

Sneddon, L.U., 2003. The bold and the shy: Individual differences in rainbow trout. J. Fish Biol. 62, 971-975. https://doi.org/10.1046/j.1095-8649.2003.00084.x.

Steffensen, J.F., 1989. Some errors in respirometry of aquatic breathers: How to avoid and correct for them. Fish Physiol. Biochem. 6, 49-59. https://doi.org/10.1007/ BF02995809.

Trenzado, C.E., Morales, A.E., de la Higuera, M., 2006. Physiological effects of crowding in rainbow trout, Oncorhynchus mykiss, selected for low and high stress responsiveness. Aquaculture 258, 583-593. https://doi.org/10.1016/j.aquaculture.2006.03. 045.

Van de Nieuwegiessen, P.G., Ramli, N.M., Knegtel, B.P.F.J.M., Verreth, J.A.J., Schrama, J.W., 2010. Coping strategies in farmed African catfish Clarias gariepinus. Does it affect their welfare? J. Fuish Biol. 76, 2486-2501. https://doi.org/10.1111/j.10958649.2010.02635.x.

Vaz-Serrano, J., Ruiz-Gomez, M.L., Gjøen, H.M., Skov, P.V., Huntingford, F.A., Øverli, Ø., Höglund, E., 2011. Consistent boldness behaviour in early emerging fry of domesticated Atlantic salmon (Salmo salar): Decoupling of behavioural and physiological traits of the proactive stress coping style. Physiol. Behav. 103, 359-364. https://doi. org/10.1016/j.physbeh.2011.02.025.

Vindas, M.A., Gorissen, M., Höglund, E., Flik, G., Tronci, V., Damsgård, B., Thörnqvist, P.O., Nilsen, T.O., Winberg, S., Øverli, Ø., Ebbesson, L.O.E., 2017. How do individuals cope with stress? Behavioural, physiological and neuronal differences between proactive and reactive coping styles in fish. J. Exp. Biol. 220, 1524-1532. https://doi. org/10.1242/jeb.153213.

Wolf, M., Van Doorn, G.S., Leimar, O., Weissing, F.J., 2007. Life-history trade-offs favour the evolution of animal personalities. Nature 447, 581-584. https://doi.org/10. 1038/nature05835. 